
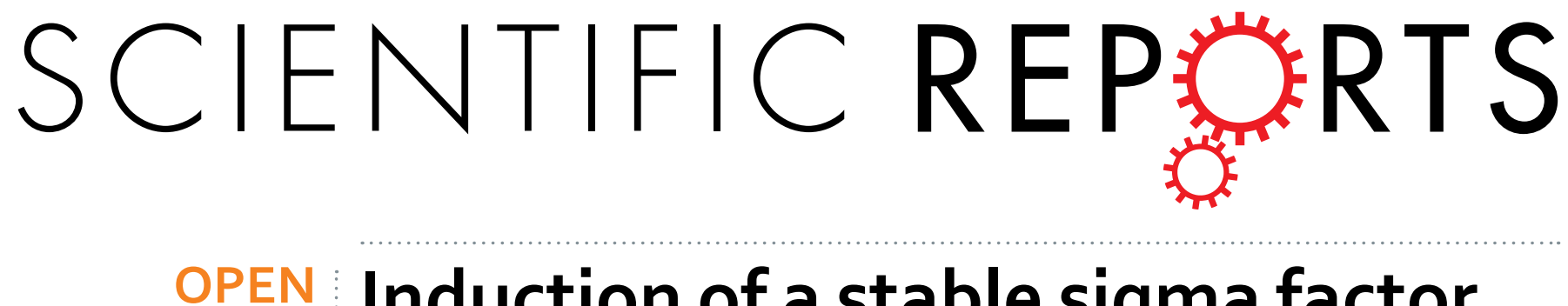

\title{
Induction of a stable sigma factor SigR by translation-inhibiting antibiotics confers resistance to
}

Received: 17 March 2016

Accepted: 06 June 2016

Published: 27 June 2016

\section{antibiotics}

\author{
Ji-Sun Yoo ${ }^{1}$, Gyeong-Seok Oh ${ }^{1}$, Sungweon Ryoo ${ }^{2}$ \& Jung-Hye Roe ${ }^{1}$
}

Antibiotic-producing streptomycetes are rich sources of resistance mechanisms against endogenous and exogenous antibiotics. An ECF sigma factor $\sigma^{R}(\mathrm{SigR})$ is known to govern the thiol-oxidative stress response in Streptomyces coelicolor. Amplification of this response is achieved by producing an unstable isoform of $\sigma^{R}$ called $\sigma^{R^{\prime}}$. In this work, we present evidence that antibiotics induce the SigR regulon via a redox-independent pathway, leading to antibiotic resistance. The translation-inhibiting antibiotics enhanced the synthesis of stable $\sigma^{R}$, eliciting a prolonged response. WbIC/WhiB7, a WhiB-like DNAbinding protein, is responsible for inducing sigRp1 transcripts encoding the stable $\sigma^{R}$. The amount of WbIC protein and its binding to the sigRp1 promoter in vivo increased upon antibiotic treatment. A similar phenomenon appears to exist in Mycobacterium tuberculosis as well. These findings reveal a novel antibiotic-induced resistance mechanism conserved among actinomycetes, and also give an explicit example of overlap in cellular damage and defense mechanisms between thiol-oxidative and anti- translational stresses.

Many actinomycetes, especially those of Streptomyces genus, are well recognized for undergoing complex developmental programs and producing diverse secondary metabolites. In soil environment where streptomycetes inhabit, thousands of bacterial species are estimated to reside in one gram of soil producing more than $10^{4}$ bioactive small molecules ${ }^{1,2}$. In natural environment, streptomycetes have to deal with numerous growth-inhibitory antibiotics which are made by themselves (endogenous) or other organisms (exogenous). Therefore, while being major producers of antibiotics, actinomycetes are the major sources of antibiotic resistance mechanisms ${ }^{3}$. The mechanisms of antibiotic resistance found in clinical pathogens derive their origin from environmental bacteria as first identified for aminoglycoside resistance in Streptomyces ${ }^{4}$. Since then, various parallel examples, such as vanHAX gene cluster for vancomycin resistance, were reported in soil actinomycetes as well as in clinical strains ${ }^{5}$.

Whether living inside the human body or in natural environment, bacteria are exposed to wide concentration ranges of antibiotics. In most cases, they are exposed to non-lethal or sub-minimal inhibitory concentration (MIC) of antibiotics. Antibiotics at sub-MIC act as signals and stressors to elicit physiological and genetic changes to cope with antibiotic stress ${ }^{6-8}$. Antibiotic resistance phenotype is induced by sub-inhibitory antibiotics through modulating gene expression and physiology (intrinsic resistance) or through changing genetic information via mutation or horizontal transfer of resistance genes (acquired resistance). Modulation of bacterial gene expression to enhance intrinsic resistance is mediated via hosts of regulators. Some known transcriptional regulators include RNA polymerase sigma factors such as $\mathrm{RpoS}^{9}$, a redox-sensitive regulator such as SoxR ${ }^{10,11}$, or a WhiB-like factor $\left(\mathrm{WblC} / \mathrm{WhiB} 7^{12,13}\right)$. Unraveling the vast array of regulatory pathways and their networks are needed to understand and control resistance mechanisms.

Among regulators that respond to environmental changes, a group of alternative sigma factors called extra-cytoplasmic function (ECF) sigma factors are abundantly encoded in bacterial genomes ${ }^{14,15}$. They are also called group 4 sigma factors consisting of only $\sigma_{2}$ and $\sigma_{4}$ domains that recognize -35 and -10 regions, respectively, of cognate promoters ${ }^{16,17}$. In Streptomyces coelicolor, 50 such factors are encoded in the genome ${ }^{18}$. Among

${ }^{1}$ Laboratory of Molecular Microbiology, School of Biological Sciences, and Institute of Microbiology, Seoul National University, Seoul 151-742, Korea. ${ }^{2}$ Korean Institute of Tuberculosis, 168-5, Osongsaengmyeong 4-ro, Osong, Cheongwon-gun, Chungcheongbuk-do, 28158, Korea. Correspondence and requests for materials should be addressed to J.-H.R. (email: jhroe@snu.ac.kr) 
A

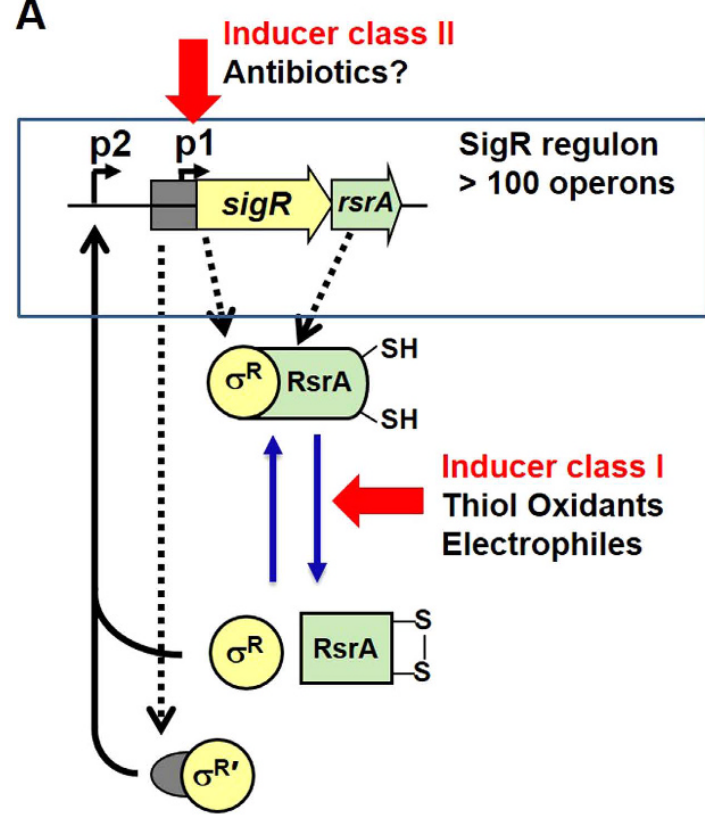

B

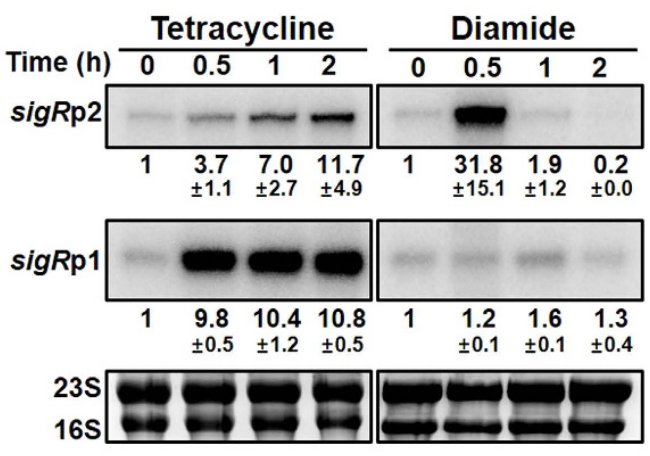

Figure 1. Induction of $\operatorname{sig} R$ transcription by antibiotics via downstream p1 promoter. (A) The regulatory loops in activating SigR regulon. Two isoforms of SigR, $\sigma^{\mathrm{R}}$ and $\sigma^{\mathrm{R}^{\prime}}$, are produced from the two promoters of $\operatorname{sig} R$ gene, sigRp1 and sigRp2, respectively. Under cytoplasmic reducing environment, the reduced RsrA binds SigR (primarily the abundant $\sigma^{\mathrm{R}}$ ), inhibiting SigR-directed transcription. Upon oxidative stress by thiol-oxidants such as diamide, di-sulfide bonds are formed in RsrA, and SigR is released from sequestration. The released SigR directs transcription of more than 100 genes (SigR regulon) that includes its own gene (from the upstream sigRp2 promoter). In contrast to $\sigma^{\mathrm{R}}$ that is very stable, $\sigma^{\mathrm{R}^{\prime}}$ with 55 more $\mathrm{N}$-terminal amino acids is very unstable. (B) Difference between induction by thiol oxidant diamide and antibiotic tetracycline. The wild-type cells were sampled at 0, 30,60 and $120 \mathrm{~min}$ after treatment with tetracycline $(2 \mu \mathrm{g} / \mathrm{ml}$, or $4.16 \mu \mathrm{M})$ or diamide $(0.5 \mathrm{mM})$ for S1 nuclease mapping of sigR-specific RNAs. The rRNA in each RNA sample were resolved in parallel. Results from three independent experiments were quantified to present values for average fold induction with standard error of the mean (s.e.m.).

them, the role of only several factors has been elucidated, such as SigR $\left(\mathrm{SCO} 5216^{19}\right), \mathrm{BldN}\left(\mathrm{SCO} 3323^{20}\right), \operatorname{SigU}$ $\left(\mathrm{SCO} 2964^{21}\right)$, SigE $\left(\mathrm{SCO} 3356^{22}\right)$, SigT $\left(\mathrm{SCO} 3892^{23}\right)$ and SigQ (SCO4908 $\left.{ }^{24}\right)$.

The SigR system in $S$. coelicolor is activated by thiol-reactive chemicals that oxidize or alkylate cysteine thiols $^{19,25}$. The induction mechanism involves the inactivation of its anti-sigma factor RsrA via forming disulfide bonds, and liberating active SigR ${ }^{26-28}$, which then positively regulates the expression of its own gene from the SigR-dependent upstream promoter (sigRp2) (Fig. 1A). The positively amplified sigRp2-derived SigR protein contains $\mathrm{N}$-terminally extended 55 more amino acid residues, and is called $\sigma^{\mathrm{R}^{\prime}}$ to distinguish it from the apparently constitutive form $\sigma^{\mathrm{R}}$ expressed from the downstream promoter $(\operatorname{sigRp} 1)^{29}$. A prominent difference between $\sigma^{\mathrm{R}}$ and $\sigma^{\mathrm{R}^{\prime}}$ is in their stability. Whereas $\sigma^{\mathrm{R}}$ is stable for hours, $\sigma^{\mathrm{R}^{\prime}}$ is short-lived with a half-life of $\sim 10 \mathrm{~min}^{29}$. Both $\sigma^{\mathrm{R}}$ and $\sigma^{\mathrm{R}^{\prime}}$ in their free state bind the core RNA polymerase and transcribe over 100 target genes to cope with the thiol-oxidative stress ${ }^{30}$. The SigR regulon includes the thiol-reducing systems which contribute to reactivating RsrA via disulfide reduction. It also includes proteases which degrade $\sigma^{\mathrm{R}^{\prime}}$, thereby turning off the response within an hour ${ }^{26,29}$. Therefore, the response of SigR-RsrA system to thiol-reactive chemical stresses is transient and is mediated by sensor RsrA and amplified $\sigma^{\mathrm{R}^{\prime}}$.

In this study, we demonstrate that multiple antibiotics induce the SigR system via yet another pathway of signal transduction, different from what conveys the thiol-perturbing signals. We show that the antibiotic induction of the SigR system proceeds via increasing the production of stable $\sigma^{\mathrm{R}}$, and this induction is mediated by WblC/ WhiB7. WblC is a WhiB-like protein conserved in actinomycetes ${ }^{31-33}$ and reported to confer resistance to antibiotics in Mycobacterium and Streptomyces ${ }^{12,34}$. WblC/WhiB7 proteins contain three functional domains such as an $\mathrm{Fe}-\mathrm{S}$ cluster binding domain with four conserved cysteines, a G(V/I)WGG turn, and an AT-hook DNA binding domain $^{35}$. The whiB7 gene is known to be induced by a variety of antibiotics via autoregulation, and WhiB7 may contribute to intrinsic resistance to antibiotics by activating antibiotic export, antibiotic inactivation and changes in thiol redox balance in mycobacteria ${ }^{13,36}$. Our work verifies sigRp1 promoter region as a novel binding site of $\mathrm{WblC/WhiB7}$ in S. coelicolor, and suggests that the expression of SigR-homologous ECF sigma factor genes (sigE and sigH) in $M$. tuberculosis may also respond to antibiotics via WhiB7.

\section{Results}

Induction of the sigR gene expression by translation-inhibiting antibiotics. While performing hygromycin-chase experiment to measure the half-life of $\sigma^{\mathrm{R}}$ and $\sigma^{\mathrm{R}^{\prime}}$ proteins, we previously observed an increase 
A

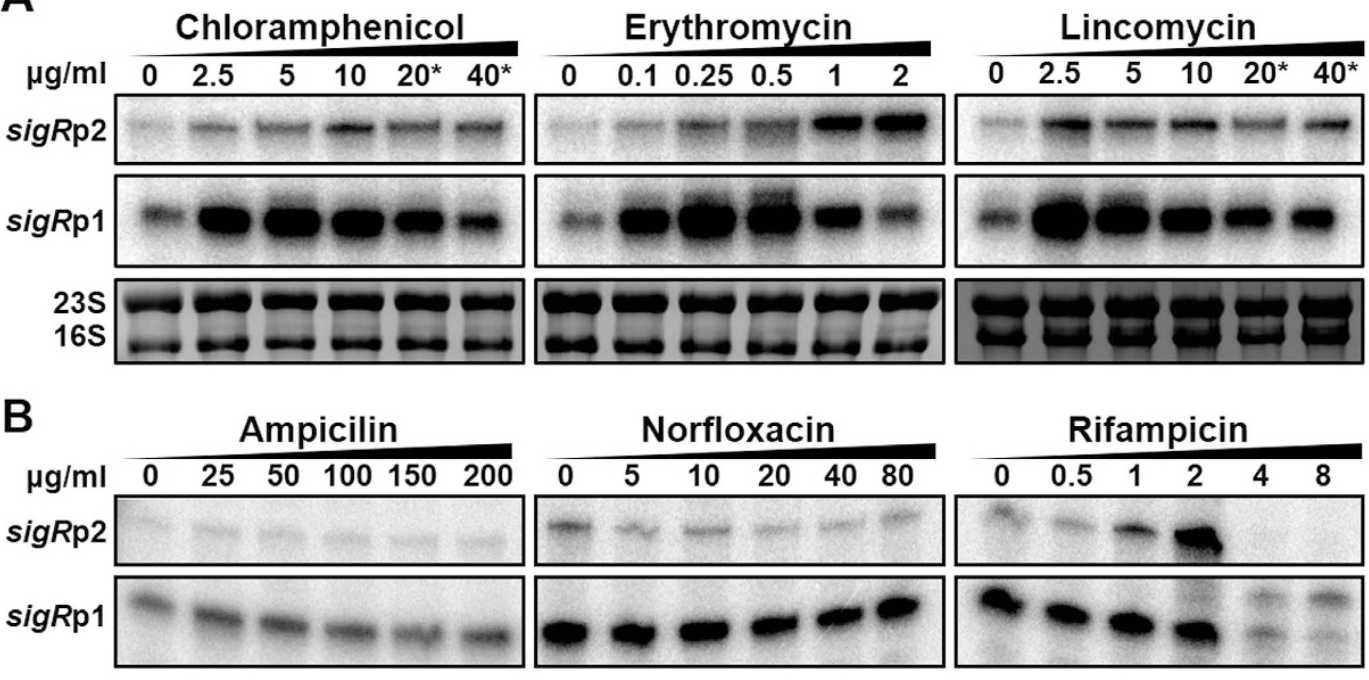

Figure 2. Translation-inhibiting antibiotics induce sigRp1 transcription. (A) Effect of antibiotics that target translation. S. coelicolor cells were sampled at $30 \mathrm{~min}$ after treatments with chloramphenicol (0 to $40 \mu \mathrm{g} / \mathrm{ml})$, erythromycin ( 0 to $2 \mu \mathrm{g} / \mathrm{ml})$, or lincomycin $(0$ to $40 \mu \mathrm{g} / \mathrm{ml})$. S1 nuclease protection assay for sigR-specific transcripts were done. The rRNA in each sample were presented as a control. The asterisk (*) denotes inhibitory concentration of the antibiotics (see Fig. S1). (B) Effect of antibiotics that target other cellular processes; cell wall synthesis (ampicillin from 0 to $200 \mu \mathrm{g} / \mathrm{ml}$ ), DNA replication (norfloxacin from 0 to $80 \mu \mathrm{g} / \mathrm{ml}$ ), and transcription (rifampicin from 0 to $8 \mu \mathrm{g} / \mathrm{ml}$ ). The sigR-specific RNA analysis was done as in panel (A).

in transcripts from the sigRp1 promoter $^{29}$. This observation was unexpected since we used to regard the $\operatorname{sig} R \mathrm{p} 1$ promoter as constitutive. We examined the effect of other antibiotics and compared it with that of thiol oxidant diamide. Figure $1 \mathrm{~B}$ shows the induction profile of $\operatorname{sigR\mathrm {p}} 1$ and $\operatorname{sigR\mathrm {p}2}$ transcripts after treatment with tetracycline $(2 \mu \mathrm{g} / \mathrm{ml})$ or diamide $(0.5 \mathrm{mM})$ for up to $2 \mathrm{~h}$. Similarly to hygromycin, sigRp1 transcripts increased significantly by about 10 -fold in response to tetracycline, in a prolonged fashion. This contrasts with the transient induction of sigRp2 transcripts by diamide as previously observed ${ }^{19,26}$. The antibiotic induction of sigRp1 transcription does not seem to be mediated by SigR itself, unlike sigRp 2 transcription, since the induction occurred even in the $\Delta s i g R$ mutant (MK1), where all transcriptions from the sigRp2 promoter disappeared (Fig. S1).

To investigate the induction of sigR mRNAs by antibiotics in further detail, we explored diverse antibiotics with different chemical structures and targets. Following 30 min treatments at varying concentrations, the sigR transcripts were monitored by S1 mapping. The results demonstrated that translation-inhibiting antibiotics such as chloramphenicol, erythromycin, and lincomycin all induced sigRp1 expression significantly (Fig. 2A). Fusidic acid and streptomycin also induced sigRp1 transcripts (data not shown). On the other hand, ampicillin, norfloxacin, and rifampicin that affects cell wall, DNA replication, and transcription, respectively, failed to increase transcripts from sigRp1 (Fig. 2B). Rifampicin induced $\operatorname{sigRp} 2$ expression at $2 \mu \mathrm{g} / \mathrm{ml}$, as observed previously in a different $S$. coelicolor strain $M 600^{37}$. Thus, the $s i g R p 1$ expression is induced specifically by translation-inhibiting antibiotics. Determination of growth inhibitory concentrations for treated antibiotics (Fig. S1) indicated that the sigRp1 induction occurred at sub-inhibitory concentrations.

Antibiotic treatment increases $\sigma^{R}$ protein and steadily induces target gene expression. Whether the increase in sigRp1 transcripts leads to increased $\sigma^{\mathrm{R}}$ protein level in the presence of translation-inhibiting antibiotics was then examined. Analytical Western blot analysis with anti-SigR antibody revealed that erythromycin $(0.25 \mu \mathrm{g} / \mathrm{ml})$ increased the level of $\sigma^{\mathrm{R}}$, but not $\sigma^{\mathrm{R}^{\prime}}$ protein, continuously for up to $2 \mathrm{~h}$ (Fig. 3A). This contrasts with the effect of thiol oxidant diamide which increased the amount of $\sigma^{\mathrm{R}^{\prime}}$ transiently by about 12 -fold, without affecting the level of $\sigma^{\mathrm{R}}$ (Fig. 3A). Parallel detection of known amounts of $\sigma^{\mathrm{R}}$ protein enabled the estimation that $\sigma^{\mathrm{R}}$ increased steadily by erythromycin to about 3 -fold level at $2 \mathrm{~h}$ after treatment compared with the untreated level. The basal amounts of $\sigma^{\mathrm{R}}$ and $\sigma^{\mathrm{R}^{\prime}}$ proteins under non-treated condition were estimated to be about $23(1.82 \mu \mathrm{M})$ and 7 $(0.56 \mu \mathrm{M})$ fmole/ $\mu \mathrm{g}$ proteins in cell extracts, respectively, assuming equal immune-specificity of $\sigma^{\mathrm{R}}$ and $\sigma^{\mathrm{R}^{\prime}}$ proteins to the antibody used. This corresponds to about 1.8 and $0.6 \mu \mathrm{M}$ in the cell for $\sigma^{\mathrm{R}}$ and $\sigma^{\mathrm{R}^{\prime}}$, respectively, assuming that about $43 \%$ of dry cell weight is from the protein, and that the wet cell weight is about 5.6 fold of the dry weight, and that cell density is $1^{38}$. Following erythromycin treatment, there appeared a non-specific band which is absent in other antibiotic-treated samples (NS in Fig. 3A). The source of this protein band is not certain, except that it is not the product of the sigR gene, since it is observed in the $\Delta$ sigR mutant after erythromycin treatment. Treatments with chloramphenicol, lincomycin, and tetracycline caused similar increase in $\sigma^{\mathrm{R}}$ without changing the amount of $\sigma^{\mathrm{R}^{\prime}}$ (Fig. 3B). No increase in $\sigma^{\mathrm{R}^{\prime}}$ by antibiotics in spite of some increase in sigRp2 transcripts could be due to the unstable nature of $\sigma^{\mathrm{R}^{\prime} 29}$.

We then examined the expression of a SigR-target gene $\operatorname{trxB}$ (SCO3890), which encodes thioredoxin reductase. Figure $3 \mathrm{C}$ shows that the SigR-dependent $\operatorname{tr} x \mathrm{Bp} 1$ transcripts increased significantly by chloramphenicol and 
A

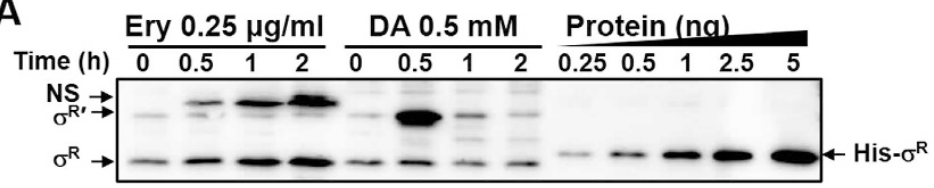

B

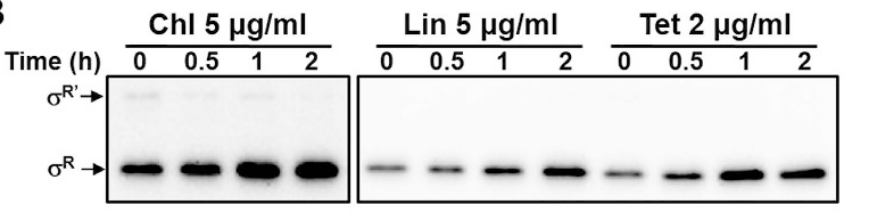

C

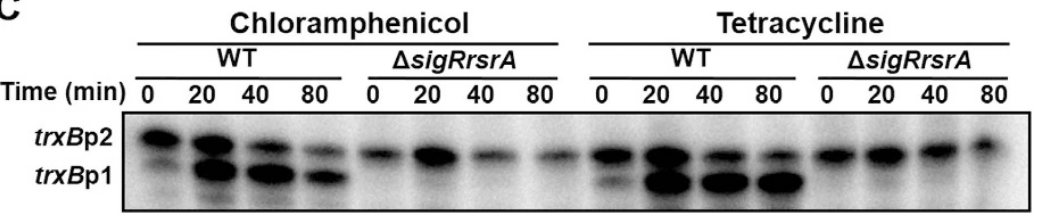

Figure 3. Steady increase in $\sigma^{\mathrm{R}}$ protein by antibiotic treatments and prolonged induction of its target promoter $(\operatorname{trx} B \mathbf{p} 1)$. (A) Steady vs. transient increase in SigR proteins by antibiotic or thiol oxidant. S. coelicolor cells were treated with either erythromycin $(0.25 \mu \mathrm{g} / \mathrm{ml})$ or diamide $(0.5 \mathrm{mM})$ for up to $2 \mathrm{~h}$, followed by western blot analysis with antibody against SigR. The positions of $\sigma^{\mathrm{R}}$ and $\sigma^{\mathrm{R}^{\prime}}$ were marked by arrows. A non-specific band (NS) produced in erythromycin-treated samples was also indicated. Analytical western blotting of indicated amounts of purified SigR (His- $\sigma^{\mathrm{R}}$; from 0.25 to $5 \mathrm{ng}$ ) with anti-SigR antibody was done in parallel to quantify the amount of SigR-specific protein bands. (B) Western blot analyses of SigR proteins following treatments with chloramphenicol, lincomycin, and tetracycline for $2 \mathrm{~h}$. (C) S1 mapping analysis of $\operatorname{tr} x B$ transcripts. The wild-type (M145) and $\Delta$ sigRrsrA (MK1) cells were treated with chloramphenicol (17 $\mu \mathrm{g} / \mathrm{ml})$ or tetracycline $(5 \mu \mathrm{g} / \mathrm{ml})$ for up to $80 \mathrm{~min}$, and analyzed for $\operatorname{tr} x B$ transcripts. The $\operatorname{tr} x B \mathrm{p} 1$ promoter is under the control of SigR.

tetracycline treatments up to $80 \mathrm{~min}$, consistent with the steady increase in $\sigma^{\mathrm{R}}$ protein. Therefore, we conclude that the translation-inhibiting antibiotics induce the production of stable $\sigma^{\mathrm{R}}$ protein, which subsequently induces its target gene expression in a prolonged fashion.

Antibiotic induction of stable $\sigma^{R}$ depends on WbIC/WhiB7. To find clues to reveal mechanisms behind antibiotic induction of sigRp1, we scrutinized its flanking sequences. One prominent feature was a stretch of AT-rich sequence, which is not common in GC-rich actinomycetous genomes, located immediately upstream of the -35 region of the sigRp1 promoter (Fig. 4A). This sequence feature is present upstream of the whiB7 promoter in Mycobacterium species, and has been proposed as the binding site of a WhiB-like (Wbl) protein WhiB $7^{36,39}$. In S. coelicolor, WblC (SCO5190) is the orthologue of WhiB7 of M. tuberculosis, and the wblC gene also has a putative auto-regulatory WblC-binding signature similarly to the whiB7 gene of M. tuberculosis (Fig. 4A). The wblC and whiB7 mutants were reported to be hypersensitive to diverse antibiotics in S. lividans, S. coelicolor, and M. tuberculosis ${ }^{12,34}$. Inspection of the promoter region of sigR-homologous genes (sigE and sigH) in M. tuberculosis $\mathrm{H} 37 \mathrm{Rv}$ also revealed the presence of putative WhiB7-binding sites immediately upstream of the promoters ${ }^{40}$ (Fig. 4A).

We investigated whether WblC is involved in inducing transcription from the sigRp1 promoter upon antibiotic treatment. The wild type and the $\Delta w b l C$ mutant cells $s^{34}$ were treated with tetracycline for up to $3 \mathrm{~h}$, and examined for sigR-specific transcripts and their protein products by S1 mapping and Western blot analyses, respectively. Results in Fig. 4B demonstrated that WblC is critically required for the antibiotic induction of sigRp1 transcription. The sigRp2 transcription, however, was induced by tetracycline regardless of the wblC mutation. Immunoblot analysis revealed that the $\sigma^{\mathrm{R}}$ protein produced from the sigRp 1 transcripts did not increase in $\Delta w b l C$ mutant, in contrast to the wild type, where $\sigma^{\mathrm{R}}$ protein increased about 2.5 -fold during the $2 \mathrm{to} 3 \mathrm{~h}$ treatments with tetracycline (Fig. 4C). These results clearly show that the increase in stable $\sigma^{\mathrm{R}}$ after antibiotic treatment depends almost entirely on WblC/WhiB7.

Antibiotics increase the amount and the binding of WbIC to sigRp1 promoter in vivo. We then investigated how WblC is involved in antibiotic induction of sigRp1 or $\sigma^{\mathrm{R}}$. For this purpose, polyclonal antibodies against $\mathrm{WblC}$ were raised in rabbits, and used to monitor WblC in cells treated with antibiotics. Figure 5A shows that the amount of WblC dramatically increased within an hour of erythromycin or tetracycline treatments. The $\mathrm{WblC}$ level decreased within $2 \mathrm{~h}$ of antibiotic treatment. The decrease at $2 \mathrm{~h}$ is more pronounced in erythromycin than tetracycline treated samples. With some slight differences in induction and shut-off kinetics, WblC was induced by other antibiotics such as hygromycin, chloramphenicol, and lincomycin to a maximal level within an hour, and then returned to the basal level within 2 or $3 \mathrm{~h}$ (Fig. 5B).

Whether WblC binds directly to the sigRp 1 promoter region in vivo was determined by chromatin immunoprecipitation (ChIP) analysis. The wild type and the $\Delta w b l C$ mutant cells were treated with tetracycline $(2 \mu \mathrm{g} / \mathrm{ml})$ 
A
S. coelicolor
M. tuberculosis
M. tuberculosis
S. coelicolor
M. tuberculosis
M. smegmatis

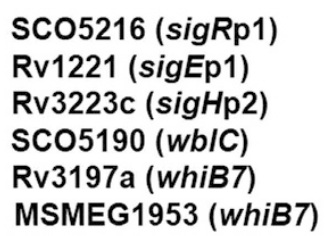

B

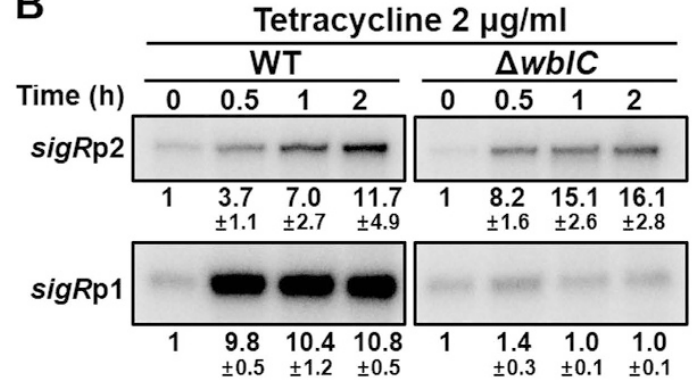

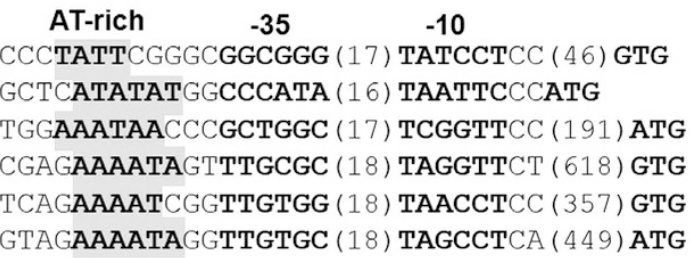

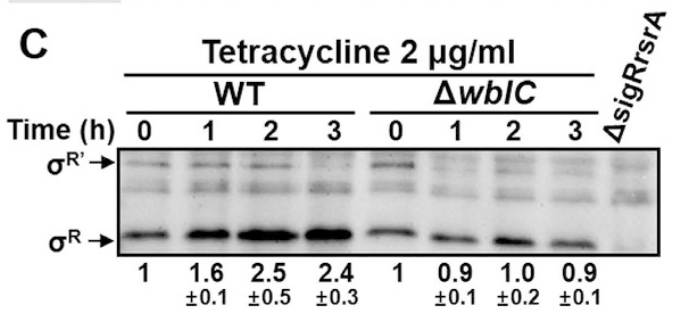

Figure 4. Antibiotic induction of sigRp1 transcription and $\sigma^{\mathrm{R}}$ production depends on WblC/WhiB7. (A) The presence of AT-rich sequence adjacent to the -35 element of promoters for sigRp1 in S. coelicolor and its homologous genes (sigE and $\operatorname{sigH}$ ) in M. tuberculosis. The AT-rich sequences upstream of the $w b l C$ in S. coelicolor, and the whiB7 promoters known to bind WhiB7 protein in mycobacteria, were also presented. (B) Antibiotic induction of sigRp1 transcripts depends on WblC/WhiB7. S1 nuclease mapping was done, following treatment of the wild type and $\Delta w b l C$ cells with $2 \mu \mathrm{g} / \mathrm{ml}$ tetracycline for up to $2 \mathrm{~h}$. Results from three independent experiments were quantified to present average values for relative fold change and s.e.m. (C) Western blot analysis of SigR proteins from cells similarly treated as in panel (B). Results from three independent experiments were quantified.

for $1 \mathrm{~h}$, followed by fixation, cell lysis, DNA shearing, and immunoprecipitation with anti-WblC antibody as described in Materials and Methods. The amount of sigRp1 promoter DNA in the precipitate was estimated by quantitative real-time PCR (qRT-PCR), along with probe sets for the upstream sigRp2 or downstream $r s r A$ regions. Figure $5 \mathrm{C}$ demonstrates that tetracycline increased $\mathrm{WblC}$ binding to the sigRp 1 promoter region (from -84 to +7 nucleotide position, relative to the transcription start site of sigRp1) by more than 10 -fold in the wild type cell, whereas no increased binding was observed in the $\Delta w b l C$ mutant. In comparison, no significant binding of $\mathrm{WblC}$ to the $\operatorname{sigR\mathrm {p}2}$ or $r s \mathrm{~A}$ regions was observed following tetracycline treatments. Therefore, we can conclude that the antibiotic treatments increase the amount of WblC, which specifically binds to the sigRp1 promoter region and mediates increased expression of $\sigma^{\mathrm{R}}$.

SigR confers resistance to translation-inhibiting antibiotics. On the basis of induction by antibiotics, we hypothesized that the $\operatorname{sigR}$ gene functions in conferring resistance to antibiotics in S. coelicolor. So far, the revealed phenotypes of $\Delta$ sigR mutant are the sensitivity to thiol oxidant diamide ${ }^{19}$, sensitivity to electrophiles (Park JH, unpublished), and increased protein aggregation in cell extracts that reflects decreased protein quality control $^{41}$. To assess antibiotic sensitivity, we spotted an equal number of spores from the wild type, $\Delta$ sigR, $\Delta w b l C$, and $\Delta s i g R$ complemented with the chromosomally integrated sigR gene, on plates containing various antibiotics. Figure 6 shows that the $\Delta s i g R$ and $\Delta w b l C$ mutations do not cause sensitivity toward non-inducing antibiotics such as ampicillin, norfloxacin, or rifampicin. However, as predicted, the $\Delta$ sigR mutant was more susceptible to inducing antibiotics such as chloramphenicol, erythromycin, lincomycin, and tetracycline. The sensitivity was restored to the wild type level by complementation with the wild type sigR gene. The $\Delta w b l C$ was more susceptible than the $\Delta s i g R$ mutant to the inducing antibiotics except chloramphenicol. These results demonstrate that the sigR gene does play a critical role in ensuring cell viability in the presence of translation-inhibiting antibiotics.

Induction of sigR-homologous genes (sigE and sigH) by antibiotics in M. tuberculosis. M. tuberculosis (Mtb) has two close homologs of SigR from S. coelicolor (ScoSigR); SigE (Rv1221; MtbSigE) and SigH (Rv3223c; MtbSigH) with $37 \%$ and $72 \%$ identity, respectively. SigH is known to regulate the thioredoxin system and heat shock proteins upon oxidative and heat stresses ${ }^{42,43}$. SigE plays a role in response to oxidative and cell envelop stresses $^{44}$. The presence of predicted WblC binding sites in the promoter regions of sigE and sigH (Fig. 4A) led us to examine the expression of these genes in Mtb upon antibiotic treatments. We treated Mtb $\mathrm{H} 37 \mathrm{Rv}$ cells with $1 \mu$ $\mathrm{g} / \mathrm{ml}$ each of erythromycin, streptomycin, or tetracycline for up to 3 days. Figure 7 demonstrates the results of S1 nuclease mapping of transcripts from the $\operatorname{sig} E$ (panel A) and sigH (panel B) genes. For Mtb_sigE gene, we detected transcripts from the two promoters (transcription start sites) as have been reported ${ }^{40}$. The sigEp1 promoter contains the WhiB7-binding motif and produces leaderless mRNA (Fig. 4A). The upstream promoter sigEp2 does not have WhiB7-binding motif but contains the promoter sequence feature recognizable by MtbSigE or MtbSigH ${ }^{45}$. We found that the sigEp1 transcripts increased significantly by all three antibiotics (Fig. 7A). The sigEp2 transcripts increased also by antibiotic treatments, but by less pronounced fold of induction. For Mtb_sigH gene, 
A

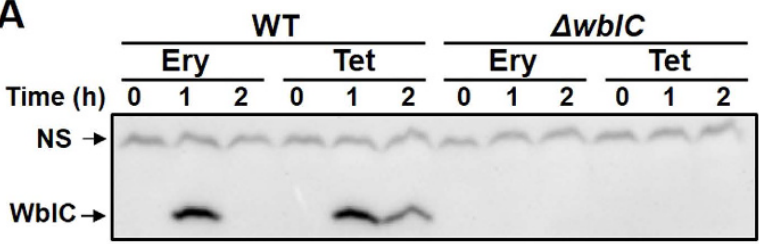

B
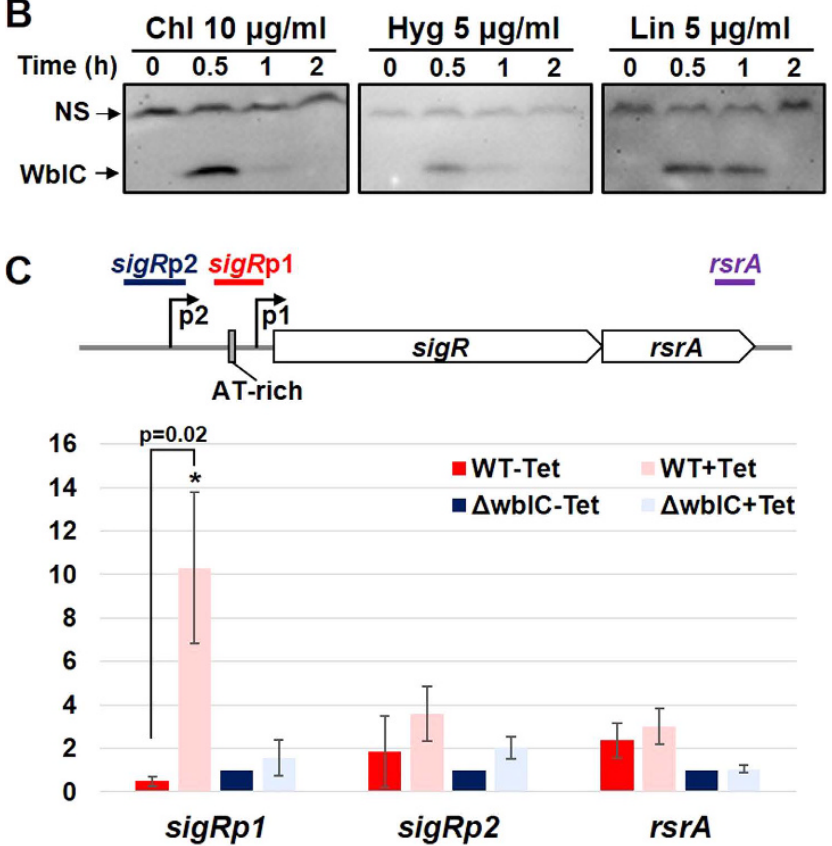

Figure 5. Increase in the amount of $\mathrm{WblC}$ protein and its binding to the sigRp1 promoter in vivo upon antibiotic treatments. (A) Western blot analysis of WblC. The wild-type and $\Delta w b l C$ cells were sampled after antibiotic treatments; Ery for $0.25 \mu \mathrm{g} / \mathrm{ml}$ erythromycin, and Tet for $2 \mu \mathrm{g} / \mathrm{ml}$ tetracycline. The WblC-specific band was detected slightly below the $15 \mathrm{kDa}$ marker, coinciding with its predicted size $(13.2 \mathrm{kDa})$. NS denotes non-specific band. (B) Western blot analysis of WblC in wild type cells treated with other translation-inhibiting antibiotics; Chl for $10 \mu \mathrm{g} / \mathrm{ml}$ chloramphenicol, Hyg for $5 \mu \mathrm{g} / \mathrm{ml}$ hygromycin, and Lin for $5 \mu \mathrm{g} / \mathrm{ml} \mathrm{lincomycin}$. (C) Chromatin immunoprecipitation with anti-WblC polyclonal antibody followed by q-PCR with genespecific primer sets for sigRp1, sigRp2, and $r s r A$ genes. The wild-type and $\Delta$ wblC cells were processed for immunoprecipitation after $1 \mathrm{~h}$ treatment with or without $2 \mu \mathrm{g} / \mathrm{ml}$ tetracycline. The enrichment of each region was estimated by quantitative real-time PCR. The relative average fold with s.e.m. were presented (y-axis), by taking the value for untreated $\Delta$ wblC sample as 1 . The asterisk $\left(^{*}\right)$ denotes $\mathrm{p} \leq 0.05$ by Student's $\mathrm{t}$-test $(\mathrm{n}=3)$.

we detected transcripts from two promoters; one from the downstream sigHp1 as reported previously ${ }^{40}$ and the other from the upstream sigHp2 recognizable by $\mathrm{MtbSigH}^{42,46}$. A WhiB7-binding motif is present in the sigHp2 promoter (Fig. 4A). Results in Fig. 7B show that both sigHp1 and sigHp2 transcripts increased by antibiotics, even though not as much as the sigE transcripts. Based on these observations, we can predict that similar pathways of upregulating SigR-like sigma factors by antibiotics are present in M. tuberculosis, and MtbSigE may play a more significant role in orchestrating response against translational blocking antibiotics.

\section{Discussion}

In this work, we demonstrated that the sigR gene expression is induced by translation-inhibiting antibiotics to produce a stable isoform of SigR, $\sigma^{\mathrm{R}}$, which elevates its target gene expression for a prolonged period, in contrast to a transient induction of $\sigma^{\mathrm{R}^{\prime}}$ by thiol-oxidative stresses. We also found that the sigR gene confers resistance to these inducing antibiotics. Previously, we identified 108 direct target genes of SigR by using ChIP-chip analysis ${ }^{30}$. Since the ChIP experiment was done after diamide treatment for $30 \mathrm{~min}$, when the majority of the sigR gene product was $\sigma^{R^{\prime}}$ (more than $80 \%$ of the total SigR; Fig. 3A), the SigR regulon we determined reflects primarily the promoters preferentially bound by $\sigma^{\mathrm{R}^{\prime}}$. Since $\sigma^{\mathrm{R}^{\prime}}$ differs from $\sigma^{\mathrm{R}^{\prime}}$ only by the $\mathrm{N}$-terminal 55 amino acids, which may not affect promoter recognition, we consider the $\sigma^{\mathrm{R}^{\prime}}$-bound genes may not differ from $\sigma^{\mathrm{R}}$-binding genes. Quite a number of SigR-target genes encode functions for thiol redox homeostasis, proteolysis, and ribosome modulation $^{30,41}$.

Treatment with translation-inhibiting antibiotics will not only slow down the synthesis of new proteins, but also result in misfolded protein products due to mistranslation or protein truncation ${ }^{47,48}$. Stalled ribosomes uncoupled with transcription can cause mRNA cleavage, resulting in ribosome stuck at non-stop mRNA, which 


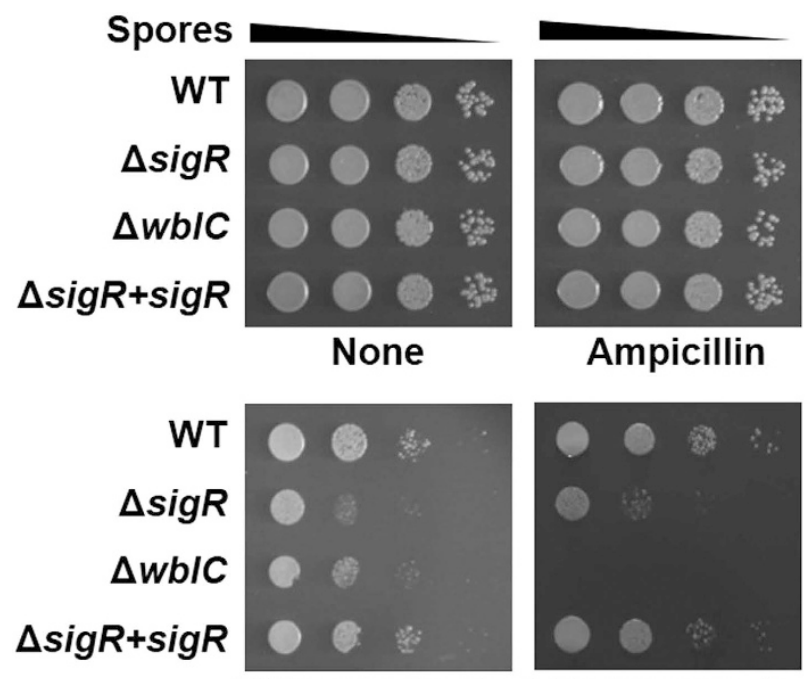

Chloramphenicol Erythromycin

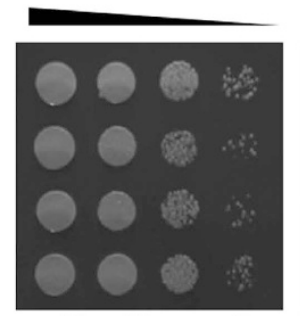

Norfloxacin

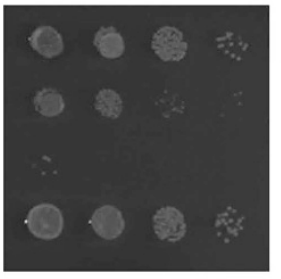

Lincomycin

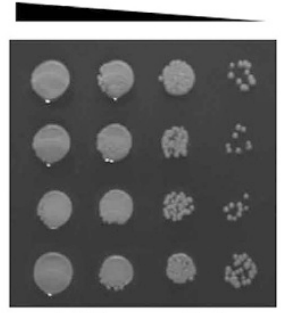

Rifampicin

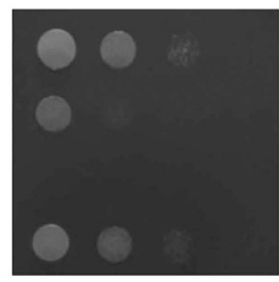

Tetracycline

Figure 6. SigR confers resistance to translation-inhibiting antibiotics. An equal number of spores of the wild-type, $\Delta \operatorname{sig} R, \Delta w b l C$, and $\Delta s i g R+\operatorname{sig} R$ complemented strains were serially diluted by 10 -fold and spotted on NA plates with or without antibiotics. Concentrations of antibiotics in the plates were $20 \mu \mathrm{g} / \mathrm{ml}$ ampicillin, $1 \mu \mathrm{g} / \mathrm{ml}$ norfloxacin, $2 \mu \mathrm{g} / \mathrm{ml}$ rifampicin, $10 \mu \mathrm{g} / \mathrm{ml}$ chloramphenicol, $2 \mu \mathrm{g} / \mathrm{ml}$ erythromycin, $10 \mu \mathrm{g} / \mathrm{ml}$ lincomycin, or $2 \mu \mathrm{g} / \mathrm{ml}$ tetracycline. Plates were incubated for 40 to $72 \mathrm{~h}$.

\begin{tabular}{|c|c|c|c|c|c|c|}
\hline A & & $24 \mathrm{~h}$ & & $48 \mathrm{~h}$ & & $72 \mathrm{~h}$ \\
\hline & - & Ery Str Tet & - & Ery Str Tet & - & Ery Str Tet \\
\hline Mtb_sigEp2 & & ment ver & $=$ & wot $=10$ & 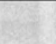 & $-=$ \\
\hline & 1 & $\begin{array}{ccc}2.1 & 1.4 & 2.6 \\
\pm 0.3 & \pm 0.1 & \pm 0.8\end{array}$ & 1 & $\begin{array}{ccc}1.8 & 2.0 & 2.1 \\
\pm 0.3 & \pm 0.4 & \pm 0.6\end{array}$ & 1 & $\begin{array}{ccc}1.3 & 7.5 & 2.7 \\
\pm 0.2 & \pm 1.4 & \pm 0.7\end{array}$ \\
\hline b_sigEp1* & & & & & & \\
\hline & 1 & $\begin{array}{ccc}2.8 & 1.6 & 5.8 \\
\pm 0.7 & \pm 0.3 & \pm 1.7\end{array}$ & 1 & $\begin{array}{ccc}2.4 & 1.9 & 3.3 \\
\pm 0.2 & \pm 0.4 & \pm 0.7\end{array}$ & 1 & $\begin{array}{r}2.310 .114 .5 \\
\pm 0.6 \pm 2.3 \pm 1.2\end{array}$ \\
\hline B & & $24 \mathrm{~h}$ & & $48 \mathrm{~h}$ & & $72 \mathrm{~h}$ \\
\hline & - & Ery Str Tet & - & Ery Str Tet & - & Ery Str Tet \\
\hline Mtb_sigHp2* & - & $m-m$ & -1 & $-\cdots$ & ent & 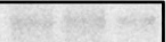 \\
\hline & 1 & $\begin{array}{ccc}0.9 & 1.1 & 2.1 \\
\pm 0.2 & \pm 0.2 & \pm 0.6\end{array}$ & & $\begin{array}{ccc}1.9 & 1.6 & 2.0 \\
\pm 0.4 & \pm 0.5 & \pm 0.4\end{array}$ & 1 & $\begin{array}{ccc}1.4 & 1.8 & 1.9 \\
\pm 0.3 & \pm 1.0 & \pm 0.7\end{array}$ \\
\hline Mtb_sigHp1 & 4 & $0 \ln m$ & 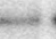 & $-\infty-\infty$ & 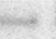 & $x+2=$ \\
\hline & 1 & $\begin{array}{ccc}1.2 & 1.9 & 3.2 \\
\pm 0.4 & \pm 0.4 & \pm 0.7\end{array}$ & & $\begin{array}{ccc}1.5 & 2.6 & 2.1 \\
\pm 0.4 & \pm 1.1 & \pm 0.4\end{array}$ & 1 & $\begin{array}{ccc}1.1 & 2.3 & 1.3 \\
\pm 0.3 & \pm 1.0 & \pm 0.6\end{array}$ \\
\hline
\end{tabular}

Figure 7. Induction of sigR-homologous gene (sigE and sigH) expression by antibiotics in M. tuberculosis (Mtb). Transcripts from the sigE panel (A) and sigH panel (B) genes of $M t b \mathrm{H} 37 \mathrm{Rv}$ strain were analyzed by S1 mapping. $M t b$ RNAs were obtained from cells grown in Middlebrooks 7H9 broth, and either non-treated or treated with $1 \mu \mathrm{g} / \mathrm{ml}$ of antibiotics for 24,48 , and $72 \mathrm{~h}$ : Ery, erythromycin; Str, streptomycin; Tet, tetracycline. Results from more than four independent experiments $(\mathrm{n}=4$ for $\operatorname{sig} E$ and $\mathrm{n}=6$ for $\operatorname{sigH}$ ) were quantified to estimate changes in the level of transcripts, taking the level of untreated sample as 1 . The average fold changes with s.e.m. were presented for transcripts from the p1 (downstream) and p2 (upstream) promoters of sigE and sigH genes. The sigEp1 and sigHp2 promoters (marked with ${ }^{*}$ ) contain putative WhiB7 binding motifs.

produces non-functional truncated protein upon ribosome rescue $\mathrm{e}^{49,50}$. Therefore, the cellular damages caused by thiol-disturbing oxidative stress can overlap with those by translation-inhibiting antibiotics to quite an extent. In light of this, the functions of predicted ribosome-associated proteins of SigR regulon such as tmRNA (ssrA), RelA, HflX, peptide-releasing factor PrfA, EngA, and ObgE need be further investigated ${ }^{30}$.

Then, why is prolonged induction of SigR required to cope with antibiotics, whereas transient induction is sufficient to cope with oxidative stress? Our results implicate that $S$. coelicolor takes longer time to overcome antibiotic stress than thiol-oxidative stress. Thiol oxidants and electrophiles that elicit thiol-oxidative stress are efficiently removed in Streptomyces by mycothiol a functional equivalent of glutathione in actinomycetes ${ }^{25}$. Increased production and recycle of mycothiol, along with increased thiol-reducing systems, after thiol-oxidative stress will 
efficiently remove chemical stressors and return the thiol redox environment back to normal in a relatively short period of time. On the contrary, the antibiotics that bind to the ribosome is harder to be cleared from the cell, affecting cell physiology for longer period of time ${ }^{51}$. This may necessitate the utilization of stable regulator, such as stable $\sigma^{\mathrm{R}}$, that can carry out the response for prolonged period of time.

We observed that the antibiotics that induced sigRp 1 transcription also induced sigRp2 transcription, even though to a lesser extent (Figs 1B,2A and 4B). The antibiotic induction of sigRp2 almost entirely depends on SigR, since no sigRp2 transcripts were observed in $\Delta$ sigR mutant (Fig. S1). Part of the reason that sigRp2 is induced by antibiotics is due to the secondary effect of increased $\sigma^{\mathrm{R}}$ that recognizes sigRp2. The results in Fig. 4B, which show that the sigRp2 is still induced by tetracycline in the $\Delta w b l C$ mutant is not easy to explain. In the absence of WblC, no increase in $\sigma^{\mathrm{R}}$ is observed, and therefore, the sigRp2 induction is likely to occur via the pathway of inactivating RsrA (Fig. 1A). It can be speculated that somehow the intracellular environment of $\Delta w b l C$ is more oxidized than the wild type following antibiotic treatment.

The more interesting question is how the production WblC protein is drastically elevated in the presence of translation-inhibiting antibiotics. The $w b l C / w h i B 7$ mRNA contains unusually long $5^{\prime}$ UTR with possible ORF for a small protein. This feature appears conserved across actinomycetes ${ }^{52}$, and may play some role in elevating WblC expression upon slowing down translation. There is also a possibility that the $w b l C$ gene expression partly depends on SigR, as predicted from the presence of SigR-dependent promoter sequence upstream of the $w b l C$ gene. The finding that the extent of antibiotic induction of the sigRp1 transcription in the $\Delta s i g R$ mutant reduced to about half of the wild type level supports this idea (Fig. S1). Further studies are in need to unravel the underlying mechanism.

\section{Materials and Methods}

Strains, plasmids, and growth conditions. Spores of S. coelicolor A3(2) strain M145, $\Delta$ sigRrsrA disruptant $(\mathrm{MK} 1)^{29}$ and $\Delta w b l C$ disruptant ${ }^{34}$ were inoculated in YEME liquid medium containing $5 \mathrm{mM} \mathrm{MgCl} \mathrm{mg}_{2} \bullet 6 \mathrm{H}_{2} \mathrm{O}$ and $10 \%$ sucrose, and were grown at $30{ }^{\circ} \mathrm{C}^{53}$. NA plates $(0.8 \%$ nutrient broth, $2 \%$ agar powder $)$ were used for spotting analysis. E. coli was grown in LB broth. The pSET152H plasmid and E. coli ET12567, a non-methylating strain containing pUZ8002 for donor functions, were used for complementation as recommended ${ }^{54}$. E. coli DE3/ gold strain and pET15b plasmid was used for WblC over-expression. M. tuberculosis $\mathrm{H} 37 \mathrm{Rv}$ cells were grown at $37^{\circ} \mathrm{C}$ in Middlebrooks 7H9 broth supplemented with $10 \%$ OADC and with or without antibiotics.

Antibiotics and reagents. Antibiotics were obtained from Sigma-Aldrich and Duchefa biochemie. The solutions were prepared freshly before treatments.

RNA preparation and S1 nuclease protection assay. S. coelicolor cells grown to $\mathrm{OD}_{600}$ of $0.3 \sim 0.4$ in YEME were treated with various antibiotics or $0.5 \mathrm{mM}$ diamide for $30 \sim 120 \mathrm{~min}$. Harvested cells were disrupted by sonication in Kirby mix. RNA isolation and S1 nuclease protection assay were done as described previously ${ }^{29}$. For mycobacterial RNA preparation, harvested cells were re-suspended in TRIzol ${ }^{\circledR}$ Reagent (Ambion, Life Technologies, Carlsbad, CA, USA), mixed with acid-washed 425 600 glass beads (Sigma-Aldrich G8772), and lysed using a mini-bead beater (BioSpec, Bartlesville, OK, USA). Following chloroform extraction and isopropanol precipitation, the RNA pellet was resolved in RNA-free water (Ambion, Life Technologies, Carlsbad, CA, USA). Mycobacterial RNA was analyzed by S1 nuclease protection assay as described previously ${ }^{29}$.

Immuno-blot analysis. Cell lysates were obtained by sonication, and its protein concentration was determined as described previously ${ }^{30}$. To detect SigR, cell extracts containing $25 \mu \mathrm{g}$ protein were diluted to the final concentration of $0.125 \mu \mathrm{g} / \mu \mathrm{l}$ with lysis buffer $(200 \mu \mathrm{l})$ that contains $100 \mu \mathrm{g}$ BSA to serve as a protein buffer. Aliquots of $8 \mu \mathrm{l}$ containing $1 \mu \mathrm{g}$ crude protein extract and $4 \mu \mathrm{g}$ BSA were resolved on 15\% SDS-PAGE. Immuno-detection was done by using polyclonal rabbit antibody against SigR and the anti-rabbit secondary antibody at 1:5000 dilution ratio, followed by ECL detection (Amersham Life Science). For detecting WblC, cell extracts containing $20 \mu \mathrm{g}$ protein were resolved on 15\% SDS-PAGE. Immuno-detection was done by using polyclonal rabbit antibody against WblC and the anti-rabbit secondary antibody at 1:10000 dilution ratio. All experimental protocols that involve animals were approved by and done in accordance with the guidelines by Seoul National University Institutional Animal Care and Use Committees (SNUIACUC).

Spotting assay to monitor antibiotic sensitivity. NA plates containing various antibiotics $(20 \mu \mathrm{g} / \mathrm{ml}$ ampicillin, $1 \mu \mathrm{g} / \mathrm{ml}$ norfloxacin, $2 \mu \mathrm{g} / \mathrm{ml}$ rifampicin, $10 \mu \mathrm{g} / \mathrm{ml}$ chloramphenicol, $2 \mu \mathrm{g} / \mathrm{ml}$ erythromycin, $10 \mu \mathrm{g} / \mathrm{ml}$ lincomycin, or $2 \mu \mathrm{g} / \mathrm{ml}$ tetracycline) were used to monitor sensitivity. An equal number of spores of wild type and mutant $S$. coelicolor strains were serially diluted by 10 -fold and spotted on antibiotic-containing NA plates using a 48-pin replica plater (Sigma). The spotted plates were incubated at $30^{\circ} \mathrm{C}$ for up to 3 days before taking photos.

Chromatin immuno-precipitation. Exponentially grown cells (at $\mathrm{OD}_{600}$ of $0.3 \sim 0.4$ ) were treated with $2 \mu$ $\mathrm{g} / \mathrm{ml}$ tetracycline for $1 \mathrm{~h}$, followed by fixation with $1 \%$ formaldehyde for $15 \mathrm{~min}$. $125 \mathrm{mM}$ glycine was subsequently added for $5 \mathrm{~min}$ at room temperature. Harvested cells were washed twice with cold TBS wash buffer $(20 \mathrm{mM}$ Tris- $\mathrm{HCl}, \mathrm{pH} 7.5,150 \mathrm{mM} \mathrm{NaCl})$. To break cells and shear DNA, cells were sonicated in RIPA buffer $(50 \mathrm{mM}$ HEPES-KOH pH 7.5, $150 \mathrm{mM} \mathrm{NaCl}, 1 \mathrm{mM}$ EDTA pH 8.0, $1 \%$ Triton X-100, 0.1\% sodium deoxycholate, $0.1 \%$ SDS, $1 \mathrm{mM}$ PMSF) with a sonicator (QSonica Q500) using a $3 \mathrm{~mm}$ tip at $30 \%$ maximum power, with 5 sec pulses for 15 times on ice. Following centrifugation at $13000 \mathrm{rpm}$ and $4{ }^{\circ} \mathrm{C}$ for $10 \mathrm{~min}$ to clear the cell debris, $50 \mu \mathrm{l} \mathrm{of} \mathrm{each}$ supernatant was set aside for input DNA control. To the cleared supernatant anti-WblC polyclonal rabbit antibody $(5 \mu \mathrm{l})$ was added, and incubated at $4^{\circ} \mathrm{C}$ for $1 \mathrm{~h}$, with gentle mixing by rotation. Subsequently, $20 \mu \mathrm{l}$ protein $\mathrm{A} / \mathrm{G}$ beads (Santacruz) and $2 \mu \mathrm{g}$ BSA were added and rotated overnight at $4^{\circ} \mathrm{C}$. The samples were centrifuged for $1 \mathrm{~min}$ at $4^{\circ} \mathrm{C}$ and $3000 \mathrm{rpm}$ and the pellets were washed once with low salt wash buffer (50 mM HEPES-KOH, 
pH 7.5, $150 \mathrm{mM} \mathrm{NaCl}, 1 \mathrm{mM}$ EDTA, 1\% Triton X-100, 0.1\% sodium deoxycholate), once with high salt wash buffer (50 mM HEPES-KOH, pH 7.5, $500 \mathrm{mM} \mathrm{NaCl}, 1 \mathrm{mM}$ EDTA, $1 \%$ Triton X-100, 0.1\% sodium deoxycholate), once with $\mathrm{LiCl}$ wash buffer $(10 \mathrm{mM}$ Tris- $\mathrm{HCl}, \mathrm{pH} 8.0,250 \mathrm{mM} \mathrm{LiCl}, 1 \mathrm{mM}$ EDTA, $1 \%$ NP-40, $1 \%$ sodium deoxycholate), and twice with TE buffer (10 mM Tris-HCl, pH 8.0, $1 \mathrm{mM}$ EDTA). DNA was eluted by incubation in the elution buffer (10 mM Tris- $\mathrm{HCl}, \mathrm{pH} 8.0,250 \mathrm{mM} \mathrm{NaCl}, 1 \mathrm{mM}$ EDTA, $1 \%$ SDS $)$ at $65^{\circ} \mathrm{C}$ for $30 \mathrm{~min}$, followed by treatment with $5 \mu \mathrm{g}$ proteinase $\mathrm{K}$ and $2 \mu \mathrm{g} \mathrm{RNaseA}$ for $1 \mathrm{~h}$ at $45^{\circ} \mathrm{C}$. $\mathrm{NaCl}$ was added to final concentration of $350 \mathrm{mM}$, and incubation continued at $65^{\circ} \mathrm{C}$ overnight for reverse-crosslinking. DNA was purified by phenol-chloroform extraction. The amount of sigRp1, sigRp2, and $r s r A$-specific DNA was quantified by qPCR (Agilent Stratagene Mx3000P), using primer sets which encompass the sigRp1 promoter region (from - 125 to $-34 \mathrm{nt}$ position, relative to the $\operatorname{sig} R$ start codon), sigRp2 promoter region (from -311 to $-186 \mathrm{nt}$ position, relative to the $s i g R$ start codon), and $r s r A$ (from +920 to +991 nt position, relative to the sigR start codon).

\section{References}

1. Schloss, P. D. \& Handelsman, J. Toward a census of bacteria in soil. PLoS Comput Biol. 2, e92 (2006).

2. Wright, G. D. Antibiotic resistance in the environment: a link to the clinic? Curr Opin Microbiol. 13, 589-594 (2010).

3. Davies, J. \& Davies, D. Origins and evolution of antibiotic resistance. Microbiol Mol Biol Rev. 74, 417-433 (2010).

4. Benveniste, R. \& Davies, J. Aminoglycoside antibiotic-inactivating enzymes in actinomycetes similar to those present in clinical isolates of antibiotic-resistant bacteria. Proc Natl Acad Sci USA 70, 2276-2280 (1973).

5. Marshall, C. G., Broadhead, G., Leskiw, B. K. \& Wright, G. D. D-Ala-D-Ala ligases from glycopeptide antibiotic-producing organisms are highly homologous to the enterococcal vancomycin-resistance ligases VanA and VanB. Proc Natl Acad Sci USA 94, 6480-6483 (1997).

6. Davies, J., Spiegelman, G. B. \& Yim, G. The world of subinhibitory antibiotic concentrations. Curr Opin Microbiol. 9, 445-453 (2006).

7. Bernier, S. P. \& Surette, M. G. Concentration-dependent activity of antibiotics in natural environments. Front Microbiol. 4, 20 (2013).

8. Andersson, D. I. \& Hughes, D. Microbiological effects of sublethal levels of antibiotics. Nat Rev Microbiol. 12, 465-478 (2014).

9. Gutierrez, A. et al. beta-Lactam antibiotics promote bacterial mutagenesis via an RpoS-mediated reduction in replication fidelity. Nat Commun. 4, 1610 (2013).

10. Dietrich, L. E., Teal, T. K., Price-Whelan, A. \& Newman, D. K. Redox-active antibiotics control gene expression and community behavior in divergent bacteria. Science 321, 1203-1206 (2008).

11. Lee, J. H., Lee, K. L., Yeo, W. S., Park, S. J. \& Roe, J. H. SoxRS-mediated lipopolysaccharide modification enhances resistance against multiple drugs in Escherichia coli. J Bacteriol. 191, 4441-4450 (2009).

12. Morris, R. P. et al. Ancestral antibiotic resistance in Mycobacterium tuberculosis. Proc Natl Acad Sci USA 102, 12200-12205 (2005).

13. Nguyen, L. \& Thompson, C. J. Foundations of antibiotic resistance in bacterial physiology: the mycobacterial paradigm. Trends Microbiol. 14, 304-312 (2006).

14. Mascher, T. Signaling diversity and evolution of extracytoplasmic function (ECF) sigma factors. Curr Opin Microbiol. 16, 148-155 (2013).

15. Staron, A. et al. The third pillar of bacterial signal transduction: classification of the extracytoplasmic function (ECF) sigma factor protein family. Mol Microbiol. 74, 557-581 (2009).

16. Lonetto, M. A., Brown, K. L., Rudd, K. E. \& Buttner, M. J. Analysis of the Streptomyces coelicolor sigE gene reveals the existence of a subfamily of eubacterial RNA polymerase sigma factors involved in the regulation of extracytoplasmic functions. Proc Natl Acad Sci USA 91, 7573-7577 (1994).

17. Helmann, J. D. The extracytoplasmic function (ECF) sigma factors. Adv Microb Physiol. 46, 47-110 (2002).

18. Hahn, M. Y., Bae, J. B., Park, J. H. \& Roe, J. H. Isolation and characterization of Streptomyces coelicolor RNA polymerase, its sigma, and antisigma factors. Methods Enzymol. 370, 73-82 (2003).

19. Paget, M. S., Kang, J. G., Roe, J. H. \& Buttner, M. J. sigmaR, an RNA polymerase sigma factor that modulates expression of the thioredoxin system in response to oxidative stress in Streptomyces coelicolor A3(2). EMBO J. 17, 5776-5782 (1998).

20. Bibb, M. J., Molle, V. \& Buttner, M. J. sigma(BldN), an extracytoplasmic function RNA polymerase sigma factor required for aerial mycelium formation in Streptomyces coelicolor A3(2). J Bacteriol. 182, 4606-4616 (2000).

21. Gehring, A. M., Yoo, N. J. \& Losick, R. RNA polymerase sigma factor that blocks morphological differentiation by Streptomyces coelicolor. J Bacteriol. 183, 5991-5996 (2001).

22. Paget, M. S., Chamberlin, L., Atrih, A., Foster, S. J. \& Buttner, M. J. Evidence that the extracytoplasmic function sigma factor sigmaE is required for normal cell wall structure in Streptomyces coelicolor A3(2). J Bacteriol. 181, 204-211 (1999).

23. Mao, X. M. et al. Dual positive feedback regulation of protein degradation of an extra-cytoplasmic function sigma factor for cell differentiation in Streptomyces coelicolor. J Biol Chem. 288, 31217-31228 (2013).

24. Shu, D. et al. afsQ1-Q2-sigQ is a pleiotropic but conditionally required signal transduction system for both secondary metabolism and morphological development in Streptomyces coelicolor. Appl Microbiol Biotechnol. 81, 1149-1160 (2009).

25. Park, J. H. \& Roe, J. H. Mycothiol regulates and is regulated by a thiol-specific antisigma factor RsrA and sigma(R) in Streptomyces coelicolor. Mol Microbiol. 68, 861-870 (2008).

26. Kang, J. G. et al. RsrA, an anti-sigma factor regulated by redox change. EMBO J. 18, 4292-4298 (1999).

27. Li, W. et al. The Role of zinc in the disulphide stress-regulated anti-sigma factor RsrA from Streptomyces coelicolor. J Mol Biol. 333, 461-472 (2003).

28. Bae, J. B., Park, J. H., Hahn, M. Y., Kim, M. S. \& Roe, J. H. Redox-dependent changes in RsrA, an anti-sigma factor in Streptomyces coelicolor: zinc release and disulfide bond formation. J Mol Biol. 335, 425-435 (2004).

29. Kim, M. S., Hahn, M. Y., Cho, Y., Cho, S. N. \& Roe, J. H. Positive and negative feedback regulatory loops of thiol-oxidative stress response mediated by an unstable isoform of sigmaR in actinomycetes. Mol Microbiol. 73, 815-825 (2009).

30. Kim, M. S. et al. Conservation of thiol-oxidative stress responses regulated by SigR orthologues in actinomycetes. Mol Microbiol. 85, 326-344 (2012)

31. Chandra, G. \& Chater, K. F. Developmental biology of Streptomyces from the perspective of 100 actinobacterial genome sequences. FEMS Microbiol Rev. 38, 345-379 (2014).

32. Soliveri, J., Vijgenboom, E., Granozzi, C., Plaskitt, K. A. \& Chater, K. F. Functional and evolutionary implications of a survey of various actinomycetes for homologues of two Streptomyces coelicolor sporulation genes. J Gen Microbiol. 139, 2569-2578 (1993).

33. Soliveri, J. A., Gomez, J., Bishai, W. R. \& Chater, K. F. Multiple paralogous genes related to the Streptomyces coelicolor developmental regulatory gene whiB are present in Streptomyces and other actinomycetes. Microbiology 146 (Pt 2), 333-343 (2000).

34. Fowler-Goldsworthy, K. et al. The actinobacteria-specific gene wblA controls major developmental transitions in Streptomyces coelicolor A3(2). Microbiology 157, 1312-1328 (2011).

35. Burian, J., Ramon-Garcia, S., Howes, C. G. \& Thompson, C. J. WhiB7, a transcriptional activator that coordinates physiology with intrinsic drug resistance in Mycobacterium tuberculosis. Expert Rev Anti Infect Ther. 10, 1037-1047 (2012).

36. Burian, J. et al. The mycobacterial transcriptional regulator whiB7 gene links redox homeostasis and intrinsic antibiotic resistance. J Biol Chem. 287, 299-310 (2012). 
37. Newell, K. V., Thomas, D. P., Brekasis, D. \& Paget, M. S. The RNA polymerase-binding protein RbpA confers basal levels of rifampicin resistance on Streptomyces coelicolor. Mol Microbiol. 60, 687-696 (2006).

38. Shahab, N., Flett, F., Oliver, S. G. \& Butler, P. R. Growth rate control of protein and nucleic acid content in Streptomyces coelicolor A3(2) and Escherichia coli B/r. Microbiology 142 (Pt 8), 1927-1935 (1996).

39. Burian, J. et al. The mycobacterial antibiotic resistance determinant WhiB7 acts as a transcriptional activator by binding the primary sigma factor SigA (RpoV). Nucleic Acids Res. 41, 10062-10076 (2013).

40. Cortes, T. et al. Genome-wide mapping of transcriptional start sites defines an extensive leaderless transcriptome in Mycobacterium tuberculosis. Cell Rep. 5, 1121-1131 (2013).

41. Kallifidas, D., Thomas, D., Doughty, P. \& Paget, M. S. The sigmaR regulon of Streptomyces coelicolor A32 reveals a key role in protein quality control during disulphide stress. Microbiology 156, 1661-1672 (2010).

42. Raman, S. et al. The alternative sigma factor SigH regulates major components of oxidative and heat stress responses in Mycobacterium tuberculosis. J Bacteriol. 183, 6119-6125 (2001).

43. Sharp, J. D. et al. Comprehensive Definition of the SigH Regulon of Mycobacterium tuberculosis Reveals Transcriptional Control of Diverse Stress Responses. PLoS One 11, e0152145 (2016).

44. Manganelli, R., Voskuil, M. I., Schoolnik, G. K. \& Smith, I. The Mycobacterium tuberculosis ECF sigma factor sigmaE: role in global gene expression and survival in macrophages. Mol Microbiol. 41, 423-437 (2001).

45. Song, T., Song, S. E., Raman, S., Anaya, M. \& Husson, R. N. Critical role of a single position in the -35 element for promoter recognition by Mycobacterium tuberculosis SigE and SigH. J Bacteriol. 190, 2227-2230 (2008).

46. Fernandes, N. D. et al. A mycobacterial extracytoplasmic sigma factor involved in survival following heat shock and oxidative stress. J Bacteriol. 181, 4266-4274 (1999).

47. Ling, J. et al. Protein aggregation caused by aminoglycoside action is prevented by a hydrogen peroxide scavenger. Mol Cell 48, 713-722 (2012)

48. Kohanski, M. A., Dwyer, D. J., Wierzbowski, J., Cottarel, G. \& Collins, J. J. Mistranslation of membrane proteins and two-component system activation trigger antibiotic-mediated cell death. Cell 135, 679-690 (2008).

49. Keiler, K. C. Mechanisms of ribosome rescue in bacteria. Nat Rev Microbiol. 13, 285-297 (2015).

50. Subramaniam, A. R., Zid, B. M. \& O'Shea, E. K. An integrated approach reveals regulatory controls on bacterial translation elongation. Cell 159, 1200-1211 (2014).

51. Yonath, A. Antibiotics targeting ribosomes: resistance, selectivity, synergism and cellular regulation. Annu Rev Biochem. 74, 649-679 (2005).

52. Dinan, A. M. et al. Relaxed selection drives a noisy noncoding transcriptome in members of the Mycobacterium tuberculosis complex. Mbio. 5, e01169-14 (2014).

53. Kieser, T., Bibb, M. J., Buttner, M. J., Chater, K. F. \& Hopwood, D. A. Practical Streptomyces Genetics, 613 (John Innes Foundation, Norwich Research Park, 2000).

54. Gust, B., O'Rourke, S., Bird, N., Kieser, T. \& Chater, K. Recombineering in Streptomyces coelicolor. Norwich: The John Innes Foundation (2003).

\section{Acknowledgements}

We thank Dr. Keith Chater (John Innes Institute) for providing the wblC mutant strain. This work was supported by a grant to J.-H. Roe (2014R1A2A1A01002846) from the Ministry of Science, ICT and Future Planning. J.-S. Yoo and G.-S. Oh were supported by B.K. Plus Fellowship for Biological Sciences at Seoul National University.

\section{Author Contributions}

J.-S.Y., G.-S.O. and S.W.R. performed the experiments. J.-S.Y. and J.-H.R. conceived the work and wrote the manuscript.

\section{Additional Information}

Supplementary information accompanies this paper at http://www.nature.com/srep

Competing financial interests: The authors declare no competing financial interests.

How to cite this article: Yoo, J.-S. et al. Induction of a stable sigma factor SigR by translation-inhibiting antibiotics confers resistance to antibiotics. Sci. Rep. 6, 28628; doi: 10.1038/srep28628 (2016).

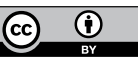

This work is licensed under a Creative Commons Attribution 4.0 International License. The images or other third party material in this article are included in the article's Creative Commons license, unless indicated otherwise in the credit line; if the material is not included under the Creative Commons license, users will need to obtain permission from the license holder to reproduce the material. To view a copy of this license, visit http://creativecommons.org/licenses/by/4.0/ 